\title{
Development of Oral Health in Africa
}

\author{
Samuel J. Thorpe \\ World Health Organization Regional Office for Africa, Harare, Zimbabwe
}

\section{Key Words \\ Poverty · Oral disease · Determinants · Strategy · Africa}

\begin{abstract}
Around $80 \%$ of African communities can be considered to be materially deprived. The presence of widespread poverty and underdevelopment in Africa means that communities are increasingly exposed to all of the major environmental determinants of oral disease. Previous approaches to oral health in Africa have failed to recognize the epidemiological priorities of the region or identify reliable and appropriate strategies to address them. Efforts have consisted of providing unplanned, ad hoc and spasmodic curative oral health services, which in most cases are poorly distributed and only reach affluent or urban communities. Realizing the limited impact of existing strategies, the World Health Organization Regional Office for Africa (WHO/AFRO) developed a regional oral health strategy to assist African countries and their partners in identifying priorities and planning preventive-oriented programmes, particularly at the district level. The long-term objective is to provide equitable and universal access to cost-effective quality oral healthcare and thereby significantly reduce the incidence of oral diseases in Africa.
\end{abstract}

Copyright $\odot 2003$ S. Karger AG, Basel

\section{Introduction}

According to the United Nations, 32 African countries are among the world's 48 least developed nations, and $80 \%$ of the people in the region fall into the low socioeconomic category [1]. Where affluence does occur, it is limited to a small urban elite whose lifestyles are similar to those in industrialized countries. The severe lack of financial and technical resources has had a direct impact on the health of the population, and while progress is being made on a number of health-related issues in Africa, the health situation in the region gives cause for concern.

The main causes of illness and death of children who survive the neonatal period include diarrhoea, acute respiratory infections, malaria, measles alone and in combination, and malnutrition. For women, the main causes of illness and death include complications associated with childbirth. For men and women, they include communicable diseases such as malaria, tuberculosis, and human immunodeficiency virus/acquired immunodeficiency syndrome (HIV/AIDS). All of these diseases are often aggravated by emergencies and disasters such as armed conflicts, drought and famine, as well as inadequate access to safe water, sanitation, nutritious foods, essential drugs, primary and secondary education, and family planning. In addition, noncommunicable diseases are emerging in Africa, especially diabetes and hypertension, due to an increase in their associated risk factors (cigarette smoking, use of alcohol, obesity and sedentary lifestyles). The presence of widespread poverty and underdevelopment in Africa means that communities are also exposed to all of the major environmental determinants of oral disease.

Dr. Samuel J. Thorp

Regional Advisor Oral Health, WHO Regional Office for Africa

PO Box BE 773, Belvedere, Harare (Zimbabwe)

Tel. +263 $4706951 / 705043$, ext. 9366, or +1 3217339366 , Fax +1 3217339009

E-Mail thorpes@whoafr.org 


\section{Current Oral Health Situation in Africa}

Dental caries and periodontal diseases are generally considered to be major oral health problems around the world. In African countries, however, they appear to be neither as common nor as severe as in the developed world. The profile of oral disease is not homogenous across Africa, with health indicators varying among countries and across groups within countries. Therefore each community needs to be individually assessed in terms of the basic epidemiological criteria of prevalence, severity (morbidity and mortality), and age-adjusted distribution in the population. Based on this form of analysis, the most severe oral health problems in Africa amongst low socioeconomic communities include cancrum oris (NOMA), acute necrotising gingivitis (ANUG), oral cancer, oral manifestations of HIV/AIDS, facial trauma, and lastly dental caries.

NOMA and the associated ANUG are still common among children in Africa. The most recent figure for the annual incidence of NOMA is 20 cases per 100,000 children aged 3-6 years. There are about 140,000 cases of NOMA each year, and tragically about $90 \%$ of these children die without receiving any care. NOMA flourishes where poverty is greatest, nutrition is poorest, and general and oral hygiene are neglected. As poverty increases and many children remain malnourished or undernourished with compromised immune systems, the prevalence of conditions such as NOMA is likely to increase [2, 3-6].

The prevalence of oral cancer is also on the increase in Africa. In developing countries, the incidence of oral and pharyngeal cancer is estimated to be 25 cases per 100,000 inhabitants. Rapid urbanization and the increasing use of tobacco and alcohol are considered to greatly increase the incidence of oral precancer [7, 8].

Africa has the highest prevalence of HIV and AIDS infections in the world. Studies have shown that oral manifestations of HIV/AIDS are very widespread, and most commonly include fungal infections such as those caused by candida, necrotizing gingivitis or oral hairy leukoplakia. In a study conducted in South Africa in 1995, 74.4\% of HIV-infected patients presented with one or more oral mucosal lesions [8-10].

Maxillo-facial trauma has increased in many countries as a result of interpersonal violence, motor vehicle accidents, and war. Chronic destructive periodontal disease is known to occur in a small proportion of most populations, regardless of location or socioeconomic status [11]. Harmful practices, such as the removal of tooth germs of deciduous canines, extraction of upper and lower anterior teeth, and the trimming or sharpening of upper anterior teeth, still prevail in Africa [5]. Fluorosis, which is more likely to occur in malnourished children, is very common in certain parts of Africa such as the Rift Valley area of East Africa [12]. Edentulism, congenital malformations and benign tumors occur, but little prevalence data is available.

According to national surveys and smaller studies the prevalence of dental caries is quite low in Africa, but there are substantial regional variations. Data on dental caries prevalence (DMFT) in children aged 12 years, available in AFRO from 39 sub-Saharan countries, show that 13 countries $(33 \%)$ have a very low DMFT $(0.0-1.1), 19$ countries (44\%) have a low DMFT (1.2-2.6), and 7 (23\%) have a moderate DMFT (2.7-4.4). About $90 \%$ of these cases represent untreated caries and the inadequacy of current oral health systems to address the problem. The situation is completely different, for example, in some Latin American countries where the DMFT among 12year-olds ranges from high to very high $(5-8)$ [8, 12-14].

\section{Determinants of Oral Health Problems in Africa}

Poverty is arguably the most important determinant of health and ill-health. The presence of widespread poverty and underdevelopment in Africa exposes communities to all of the major environmental determinants of oral disease. In a continent where the majority of the population is desperately poor, preventable oral diseases such as NOMA and oral cancer are rife. In addition, increasing urbanization has been shown to lead to observable increases in the prevalence of oral disease, and high levels of bottle feeding in cities have been associated with high rates of baby bottle tooth decay. Greater access to alcohol is associated with higher levels of interpersonal trauma and oral cancer. The African region also faces an acute lack of recent, reliable and comparable data, as well as processes for converting data into information for planning.

\section{Previous Approaches and the Response of the World Health Organization}

Previous approaches to improving oral health in Africa have been modelled on those of affluent countries and have therefore failed to recognize the epidemiological priorities of the region and identify reliable and appropriate strategies to address them. Efforts have consisted of 
providing unplanned, ad hoc and spasmodic curative oral health services, which in most cases are poorly distributed and reach only affluent or urban communities $[5,8,15]$. The main problems can be traced to the following:

- lack of national oral health policies and plans,

- inappropriately trained dentists,

- services that benefit only affluent and urban communities,

- services that are almost entirely curative,

- lack of equipment and materials, supplies, and maintenance [16].

A successful approach to oral health in the region needs to take these circumstances into account in order to effectively address the real determinants of oral disease. Prevention-oriented services need to be properly planned, administered, and evaluated, especially those that relate to participatory health education and oral health promotion. The community should be involved in identifying oral health problems, needs and interventions, and the proper balance between personnel types and population needs should be maintained.

At its 48th session in September 1998, the World Health Organization/Regional Office for Africa (WHO/ AFRO) adopted an oral health strategy for Africa for the period 1999-2008 that would assist countries in identifying priorities and planning viable programmes. The long-term vision is that all people of the region should enjoy improved levels of oral health through the realization of the following objectives:

- a significant reduction of all oral diseases in the region,

- equitable access to cost-effective quality oral healthcare,

- the adoption of healthy lifestyles [16].

To guide and sustain the effective implementation of this strategy, the following principles were adopted:

- The promotion of oral health and the prevention of oral diseases should be given high priority.

- Oral health interventions should be focused on the district and its communities.

- Interventions which have proven efficacy should be used.

- Oral health should be integrated into all public healthcare programmes.

- Communities should participate in oral health activities.

To focus the limited resources more effectively on these various priorities, the following five strategic orientations have been identified:
1 Advocacy and social mobilization: using social marketing and participatory methods to mobilise support for oral health.

2 Capacity building: developing human resources through appropriate training and retraining programmes.

3 Information, education and communication: providing appropriate information to individuals, families and communities for healthy oral health lifestyles.

4 Equitable access to quality oral health services: achieving greater equity in oral health services, particularly for rural, peri-urban and underserved communities.

5 Promotion of operational research: developing a research culture in order to encourage essential research on oral health problems and needs [16].

\section{Partnership and Coordination}

The district health management team has the primary responsibility for implementing these programmes, strategies and interventions. In order to facilitate the implementation of oral health activities and mobilise resources, partners are being identified and a wide network of interested parties is being established. Partnerships between community interest groups and health and development workers are instrumental for the successful operation of district oral health plans. At the national level, partners include professionals in commerce, industry, dentistry, medicine and allied fields, as well as professional associations, NGOs, aid agencies, WHO and other UN agencies. At the regional level, countries exchange information about their experiences in implementing oral health programmes, in the spirit of Technical Cooperation among Developing Countries (TCDC). In addition, WHO collaborating centres for oral health in the region provide expertise and resources, particularly in the areas of capacity building and research promotion [16].

Coordination among partners is crucial for the implementation of oral health programmes and extends well beyond the mere sharing of information. Where a provincial organizational level exists in a country, it has the responsibility to support district health activities and coordinate programmes that cross district boundaries, providing the link between district and national levels of activity. The provincial level helps districts with the coordination of tender processes, data collection and analysis, planning processes and resource allocation. The national level is responsible for overall coordination, as opposed to programme or service delivery, and must be properly 
equipped for this role. At the regional level, coordination is carried out by the Division of Noncommunicable Diseases, in collaboration with existing WHO structures and governing bodies [16].

\section{Conclusion}

A new way of interpreting and responding to oral health problems in Africa is long overdue. It should begin with a systematic interpretation of oral health information through the application of basic epidemiological principles at the most local level possible. Taking the unique context of each community into consideration, strategies must be built that address the social, economic and environmental circumstances that put communities at risk of ill-health, in order to limit or eradicate known determinants of oral ill-health and disease. To this end, WHO/AFRO has designed preventive programmes and new intervention strategies that aim to provide equitable and universal access to quality oral health services through the district health system. It is quite clear that the successful delivery of interventions that are affordable and effective in improving community oral health depends on the degree to which they can be integrated with general health services, and the extent to which the political, economic and professional powers that govern decision-making in health and development sectors are involved.

\section{References}

1 World Bank: World Development Report 1994. World Bank.

2 Contreras A, Falkler AW Jr Enwonwu CO, Idigbe EO, Savage KO, Afolabi MB, Onwujekwe D, Rams TE, Slots J: Human Herpesviridae in acute necrotizing ulcerative gingivitis in children in Nigeria. Oral Micrbiol Immunol 1997;12:259-265.

3 Enwonwu CO: Review of oral diseases in Africa and the influence of socioeconomic factors. Int Dent J 1981;31:29-38.

4 Enwonwu CO: Infectious oral necrosis (cancrum oris) in Nigerian children: A review. Community Dent Oral Epidemiol 1985;13. 190-194.

5 Enwonwu CO: Societal expectations of oral health: Response of the dental care system in Africa. J Publ Hlth Dent 1988;80:84-93.

6 Enwonwu CO: Noma: A neglected scourge of children in sub-Saharan Africa. Bull Wld Hlth Org 1995;73:541-545.
7 Hille JJ, Shear M, Sitas F: Age standardized incidence rates of oral cancer in South Africa, 1988-1991. J Dent Assoc S Afr 1996;51:771776.

8 Hobdell MH, Thorpe SJ: Oral health in Africa: where are we now and why is there inequity? Proceedings of the joint CDA/WHO workshop; in Myburgh NG (ed): Promoting Equity in Oral Health. Cape Town, Faculty of Dentistry and WHO Collaborating Centre, University of the Western Cape, 1996.

9 Arendorf T, Sauer G, Bredenkamp B, Cloete C: Guidelines for the Diagnosis and Management of Oral Manifestations of HIV Infection and AIDS. Cape Town, Faculty of Dentistry and WHO Collaborating Centre, University of the Western Cape, 1997.

10 Tukutuku K, Muyembe-Tamfum L, Kayembe K, Kandi K, Ntumba M: Oral manifestations of AIDS in a heterosexual population in a Zaire hospital. J Oral Pathol Med 1990;19:232-234.
11 Cuttress TW: Periodontal health and periodontal disease in young people: Global epidemiology. Int Dent J 1986;36:146-152.

12 Manji F, Mosha H, Frencken J: Tooth and surface patterns of dental caries in 12-year-old children in East Africa. Community Dent Oral Epidemiol 1986;14:99-103.

13 Manji F, Baelum V, Fejerskov O: Dental fluorosis in an area of Kenya with 2 ppm fluoride in drinking water. J Dent Res 1986;65:659-662.

14 Fejerskov O, Manji F, Baelum V: The nature and mechanisms of dental flurosis in man. $\mathrm{J}$ Dent Res 1990;69:692-700.

15 Thorpe SJ: A Regional Overview of Oral Health Services in Africa. Proceedings of the Medic Africa 1995 workshop on oral health policy; in Myburgh NG (ed): Future Directions for Oral Health in South Africa. Cape Town, Faculty of Dentistry and WHO Collaborating Centre, University of the Western Cape, 1995.

16 World Health Organization, Regional Office for Africa: Oral Health in the African Region: A Region Strategy (1999-2008), 1998. 